\title{
P03-65
}

\section{ASSESSING RESPONSE TO COGNITIVE-BEHAVIORAL GROUP THERAPY FOR PANIC DISORDER WITH COMORBID MOOD}

DISORDERS

E. Heldt, G.A. Salum, C. Blaya, L. Kipper, G.G. Manfro

Psychiatry Service - Anxiety Disorders Program, Hospital de Clínicas de Porto Alegre (HCPA), Universidade Federal do Rio Grande do Sul (UFRGS), Porto Alegre, Brazil

Objective: To evaluate the response to group cognitive-behavioral therapy (CBGT) for panic disorder (PD) in patients with and without comorbid mood disorders (MD).

Method: A total of 113 patients took part in 12 CBGT sessions for PD. The diagnoses of PD and comorbid MD (depression and/or dysthymia) were established using the Mini International Neuropsychiatric Interview (MINI). Outcome measures were assessed by Clinical Global Impression (CGI), Hamilton-Anxiety and Panic Inventory. Remission was considered when CGI $\leq 2$ and there was absence of panic attacks.

Results: One-hundred patients (91\%) completed the CBGT protocol and 37(32.7\%) patients had PD with comorbid MD. At the end of the treatment, $41(36.3 \%)$ patients met criteria for symptom remission. The remission rates were $47.4 \%$ in patients without MD versus $13.5 \%$ in patients presenting with comorbid $M D\left(R R=3.50 ; I_{95 \%}[1.5\right.$ to 8.19$\left.] ; p<0.001\right)$. Both groups showed significant improvement on all clinical outcomes throughout treatment. A differential effect was identified between those with and those without comorbid MD both in the CGI (pgroup $x$ time $=0.020$ ) and in anticipatory anxiety (pgroup $x$ time $=0.017$ ).

Conclusions: Panic patients without MD improved better to CBGT than those with MD. Maybe more sessions regarding depression should be added to the protocol in this subgroup of patients with MD. However, given that there is a general reduction of PD symptoms in all patients, CBGT must not be excluded. 